\title{
Expert Knowledge Presentation: Using concept map as a new method
}

\author{
Pei Pei ${ }^{1, a}$, Lin Zhang ${ }^{2, b}$ \\ ${ }^{12}$ School of Computer Science, Anhui Sanlian University, Anhui 230000, China \\ aEmail:elfinpeipei@sina.com \\ ${ }^{b}$ Email:sirenrabbit@sina.com
}

Keywords: Expert knowledge; Knowledge Representation; Concept map;

\begin{abstract}
Expert knowledge is very important and necessary in any field. It generally refers to the empirical experts and determine knowledge which expert used to solve problem. We can acquire unique and effective methods and knowledge systems from experts formed during their long term learning and working, so it can greatly save our time. Usually, we can acquire expert knowledge directly and indirectly but this knowledges is not comprehensive, complete and can also be difficult to presents tacit knowledge. Concept map is a tool to organize and representation knowledge. Especially, during the expert knowledge extraction it can better represent expert knowledge system and structure, promote the elicitation of expert knowledge, and improve the accuracy of the knowledge extraction.

In this paper, we review and analyze relevant literature to find the mode on how to extract expert knowledge using concept map. A model of knowledge representation is given to illustrate the whole process of extract expert knowledge using concept maps.
\end{abstract}

\section{Introduction}

Expert knowledge is refers to the empirical experts and determine knowledge which expert use to solve problem ( Liu Dayou,1988).Expert knowledge is very important and necessary in any professional field. There are a lot of reason to directly acquire knowledge from experts, rather than to discover or rediscover ourselves. First of all, it is proved that meaningful reception learning is more efficient and practical than found learning for individual (Novak \& Gowin, 1984) to acquire knowledge from experts (teachers), is an important and the most efficient form of human learning. Besides, the internal experience and wisdom succession is vital for an organization, we need timely access to and reserve the expert knowledge in the organization, maintain organizational memory, in case of expert's sudden departure. Finally, expert systems have been more and more widely used during development of computer technology and knowledge science. The most important step in building an expert system is the expert knowledge acquisition. Expert knowledge is not, however, like the textbook knowledge, can use the language or text express so clearly, and often is difficult represent (such as tacit knowledge). Therefore, how to guide the experts clearly express their inner knowledge, so that make effective communication and further use. In other words, knowledge representation techniques get more and more attention from researchers. This article first analysis the characteristics of expert knowledge, introduces the common methods of expert knowledge elicitation. Finally, analysis and provide the model of elicitation expert knowledge by concept map.

\section{Expert Knowledge.}

Definition of Expert Knowledge. Expert is a person who has superior performance in specific areas (Johnson et al.,1987).Expert knowledge generally refers to solve the problem by using the empirical, propositional knowledge. The reason why experts are experts, is because In addition to the field of public knowledge, but also including the expert knowledge of the individual, which the former accounts for $90 \%$, while the latter account for $10 \%$ of the whole expert knowledge in the expert knowledge structure 
(Huosong, Xia, 1999). The experts' exceptional performance not only depends on domain knowledge they mastered, but also rely on grasp the relation of objects in problem situation (Bradley et al., 2006).

There some other reasons to introduce expert as below: 1.experts read a large number of professional books, papers, reports, etc., in the field and therefore accumulated a lot of professional knowledge and solid background knowledge in a certain field; 2.The knowledge in the expert's mind has a certain structure, that is to say ,the knowledge architecture is not isolated or messy, but is a connect with each other, clear the relationship between the up and down, strong expanded system; 3. When expert store knowledge as well as store clues to extracted knowledge which makes knowledge extraction easy;4.experts practice more , and summarize a lot of familiar hypothesis testing model, so he can deduce unknown from known.

We may concern two aspects of expert knowledge, for one thing that is the content and storage in memory, for the other thing is that how expert use their knowledge to solve complex problem. In other words, we should not only investigate the amount and type of expert knowledge, but also analyze the methods of knowledge application.

The Characteristics of the Expert Knowledge. Bransford has summarized basic characteristics of expert knowledge as following (Bransford et al., 2000). 1. Experts can identify information characteristics and meaningful information modes than novice; 2. Experts obtained organized knowledge -- - organized with core ideas or concepts; 3.The expert's knowledge is based on conditions -- -- it includes specification in useful situations; 4. Experts can extract important details from their own knowledge flexibility; 5. Expert knowledge is adaptive knowledge, they can deal with the new situation as possible as they can by metacognition to challenge their own professional knowledge level, and try to go beyond them. These five aspects are interrelated. The core is to stress deep understanding in field knowledge which this deficiency make the essential difference between novice and expert.

Coffey et al. think: there exist two different style of expert knowledge when complete task, one is expert's memory, the other is external resource, relatively few, found in outside such as technical reports, manuals, books and online resources (Coffey et al., 2002).External resources is present explicit form, but the real skill is in the expert memory which is implicit. So we need to induce expert's knowledge into communicable explicit knowledge mode.

Ways of Expert Knowledge Extraction. In order to save our time when solve the problem , we can get the knowledge system and unique, effective solution formed from experts' long time study and work. Usually, there are two ways of knowledge extraction: direct and indirect. The direct way is to communicate with experts face, such as interviews, analysis of similar tasks or experiment. The indirect way is get to know the organized information by expert or knowledge engineers. These produce some knowledge products during the process, such as concept map, interview manuscript, video footage of a topic or a theory when expert discussed. But these expert knowledges are not complete a full , also hardly to obtain tacit knowledge hidden in experts mind (Hoffman, et al., 1995).Hoffman, et al.described varieties of extraction method, one way is called contrived techniques which will use some empirical control or half empirical control in some task expert familiar with (Hoffman, et al. , 1995). In fact, people do not simply use a single method, but will take a variety of methods integrated, adopted in different stages in combination when extract expert knowledge.

\section{Expert Knowledge Extraction by concept map.}

Concept Map. Concept map is a tool used to organize and represent knowledge. It is usually put a

Theme concepts in the circle or square, and then using lines connect with relative concepts and propositions, and put the significance of the relationship on lines between two concepts. Concepts, propositions, cross-links and hierarchical frameworks are four basic features of concepts. Concepts and propositions are presented in hierarchical relations, the most extensive meaning, the most general concept on the top of the concept map, the secondary generic concept under the hierarchy (Zhao Guoqing, etc., 2004; Novak \& Gowin, 1984). 
Using Concept Map represent Expert knowledge. A lot of knowledge is stored in the expert's mind, but in many cases, few experts also clear know how to extract and apply to the practical situation. Concept map make the tacit knowledge representative, no only can display the structure of the expert knowledge, but also can to change, complete constant. Not only others can benefit from it, the expert himself can find some of the points without ordinary.

More importantly, concept map as a kind of knowledge visualization tools, can create and promote group knowledge transfer (Zhao Guoqing, etc., 2005).It build a bridge between expert and knowledge inducer for good communication, reducing the misunderstanding and improving correction of the knowledge extraction to a great extent.

The Model of Using Concept Map Extract Expert Knowledge. Coffey et al. (2002) proposed a using concept map application model (PreSERVe) of expert knowledge. In this model, concept map is used as a method of knowledge representation. The final model including field of concept, principle, and the relations directly by the experts concluded, modified or confirmed. PreSERVe is the short of five section of extraction, they respectively are: preparation. scoping,representaition, rendering and verification. This model integrated various kinds of knowledge extract strategy and provides a principled guidance. PreSERVe model as shown in figure 1,PreSERVe- — the method of Knowledge modeling. We can black solid line means "process flow," dotted line means "resource flow ", every stages of the work is as follows (Coffey, et al., 2002).

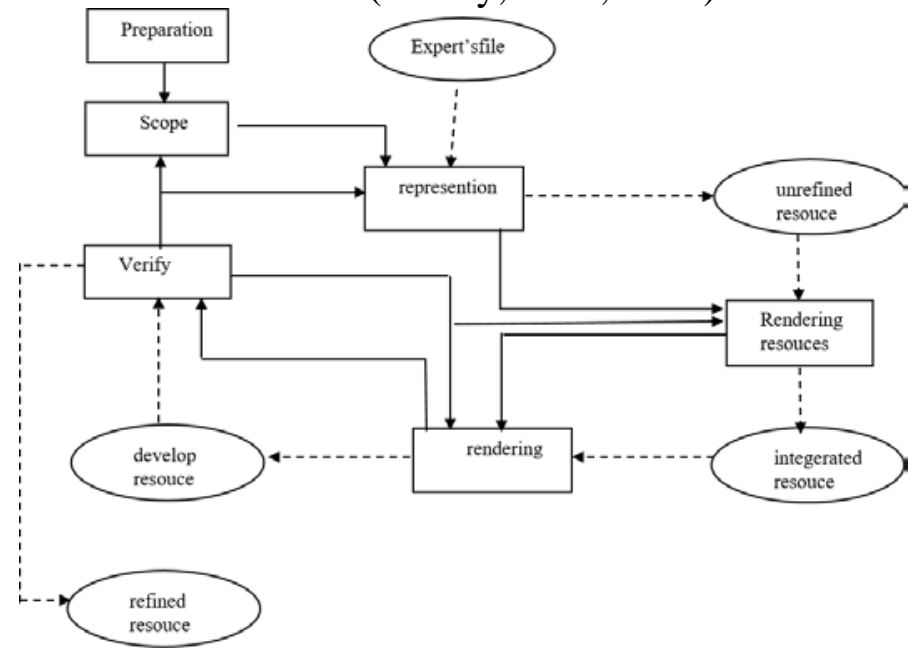

Figure 1 PreSERVe- - the method of Knowledge modeling

Preparation: Make sure the object experts and choose knowledge area. Knowledge management engineers will be arranged to learn with these expert and establish close, friendly relationship. After this mission completed, the following processes need to be repeated.

Scoping: Define the detail scope of extracted knowledge.

Extraction: Represent expert knowledge in the form of concept map by reading experts' file or face to face interview.

Rendering: Edit, modify the elements in the knowledge model, and put these elements into knowledge model.

Verification: This phrase must be confirmed both on "physical" and "concept "at the same time. Physical verification is to ensure that all elements can be in concept map, like hyperlinks can work normally, media display is correct. Concept verification (also we can say "semantics verification") is to check whether the information is complete, correct and contain the best available content. This content's structure, arrangement and retrieval mechanism is meaningful which has fill in the blank of extracted knowledge and avoid redundant. Finally, we will get a more comprehensive model due to this iterate process.

\section{A Case of Expert Knowledge Extraction Using Concept Map: Nuclear power industry expert Knowledge Capture and Represent}


Case Background. Nuclear power industry provides about $20 \%$ of energy consumption which made great contributions for home energy supply in the United States .But in recent years, the industry has became hard to absorb some talented people engaged into this the field of work. It is estimated that in the there will be about $40 \%$ of the workers reach retirement age, and the nuclear power industry will face the danger of expert knowledge loss due to experts retirement in next five years (Coffey, et al., 2004).In response to brain drain and knowledge loss, the nuclear industry launched the knowledge maintain project .This project was built for the purpose of capturing and understanding technical experts' skill, to train new hand to replace retiring senior engineers, scientists, and technical engineer, and to acquire the best knowledge practice without Document knowledge before they completely disappear. This case is knowledge retention test research project, study the knowledge extraction in the form of concept map. The study was conducted in a nuclear power station, work with an air of nuclear physics experts. During this conversation, concept map was used as a main guide to help present and understand. Besids,the experiment study includes two "two and a half days on-site interview" and a final summary to make all kinds of tangible material included. This expert of interview who has the key position is elected by the company employees, but will retire soon. When the researchers get to know more about him, they found that he is very talkative and knowledgeable and he had worked in different company, with work rich.experience.

Research Process. The first phase (before two and a half day of work) : The purpose of this phase is to produce a series concept to capture the expert's knowledge scope. The first concept map is called a " Corner stones " (means the basis of work).For this expert, the "corner stone" is nuclear physics, radiation detective, programming, and interpersonal relationship. Two informal figure are also produced, one is named " getting things done"- including operation analysis, repair equipment, impact management, etc.; The other is called "Learning as you go", emphasized the importance of lifelong learning.

The important part of this experts' work is monitoring instruments and emissions diameter. Therefore, we create various monitoring diagram, including gas, liquid, process and emissions. The theme of radioactive detection is also create into a concept map, including radiation types and detecting method. Moreover, several other concept map is mainly created to report and explain.

Data reduction is another concern of the work, so it generates a lot of calculation, statistics and diagrams of mistakes correction. Sub-knowledge can be learned from these charts.

The second stage (after two and a half days of work): The aim is to further explore and examine problem of diagram first generated. On the first day of the second stage, it generated the map of algorithm, germanium detectors, control procedures, monitoring commands, reviewed and modified the concept map identified in the first stage. This stage also produced two particularly interesting figure, because they have proved that the concept map can be used to draw out the ability scope of expert knowledge. One of them is "Activity maps", this chart is description of "what have done". This is different from the concept of noun, it contains basic activities of introduction and primary of this kind of job. At the end of the second interview, it will generate preliminary knowledge model, these models will be presented to the experts, and get confirmation.

The organization of knowledge model. The aim of this work is build an expert knowledge key model. This model needs to be able to articulate represent the expert's all aspects knowledge expert, including the organization of the relations, coherent, and navigation of concept map. The Top Map is the organized framework of various kinds of figure and it provides navigation for detail visiting in the model (part of the original node image has a hyperlink, can enter subgraph by clicking the link).Figure 2 is the top level map from the expert, it reflects the basic ability and skill and a whole process that a new person must go through in this job. 


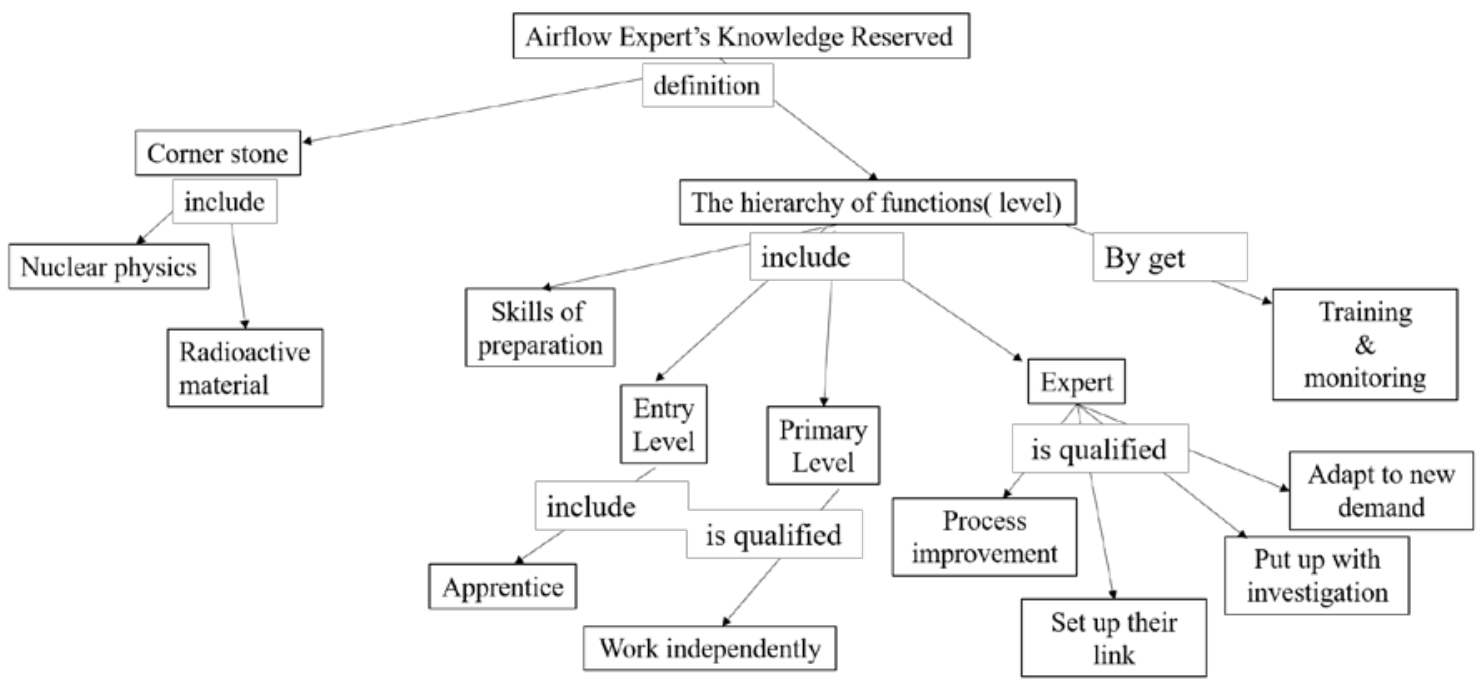

Fig 2 Top level map of airflow expert’s knowledge

Click on the left child (Cornerstones) of the root node of "top level map" can go to see detail charts.Fig. 3 is a typical example of detail representation using concept map.It covers necessary job skills assessment by the expert, it can also be linked to other detail through hyperlinks such as radiation detection, software, statistics, etc.

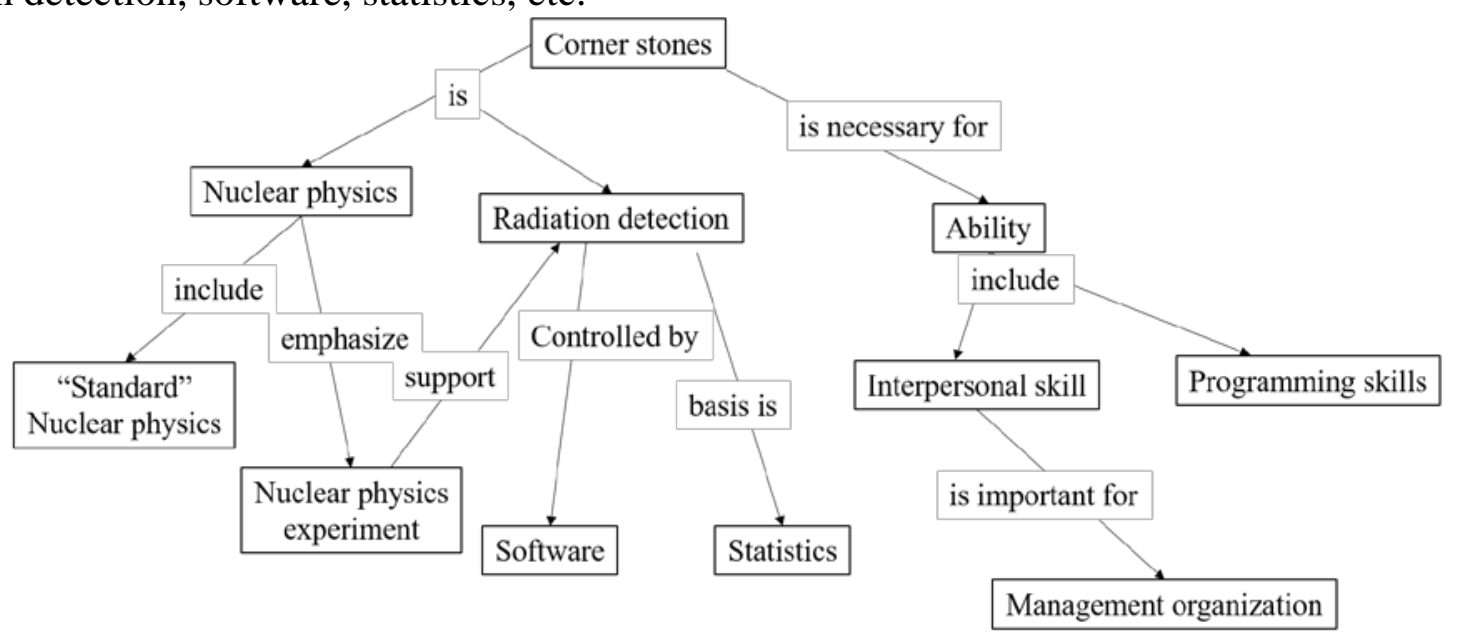

Fig.3 “Corner stones” level map

Due to the project requires people who in charge of knowledge represetation,capture experts' necessary knowledge and skills,but there is an obvious gap in the process. That is: Although expert knowledge and skills to rely on to this work has listed, but it does not capture the specific behavior based on these skills and knowledge and that make knowledge representation people create activity diagram.In this diagram, concept is related to basic activities of work,the conjunctions describes the relationship between these activities.Fig. 4 is an example of basic activities diagram. 


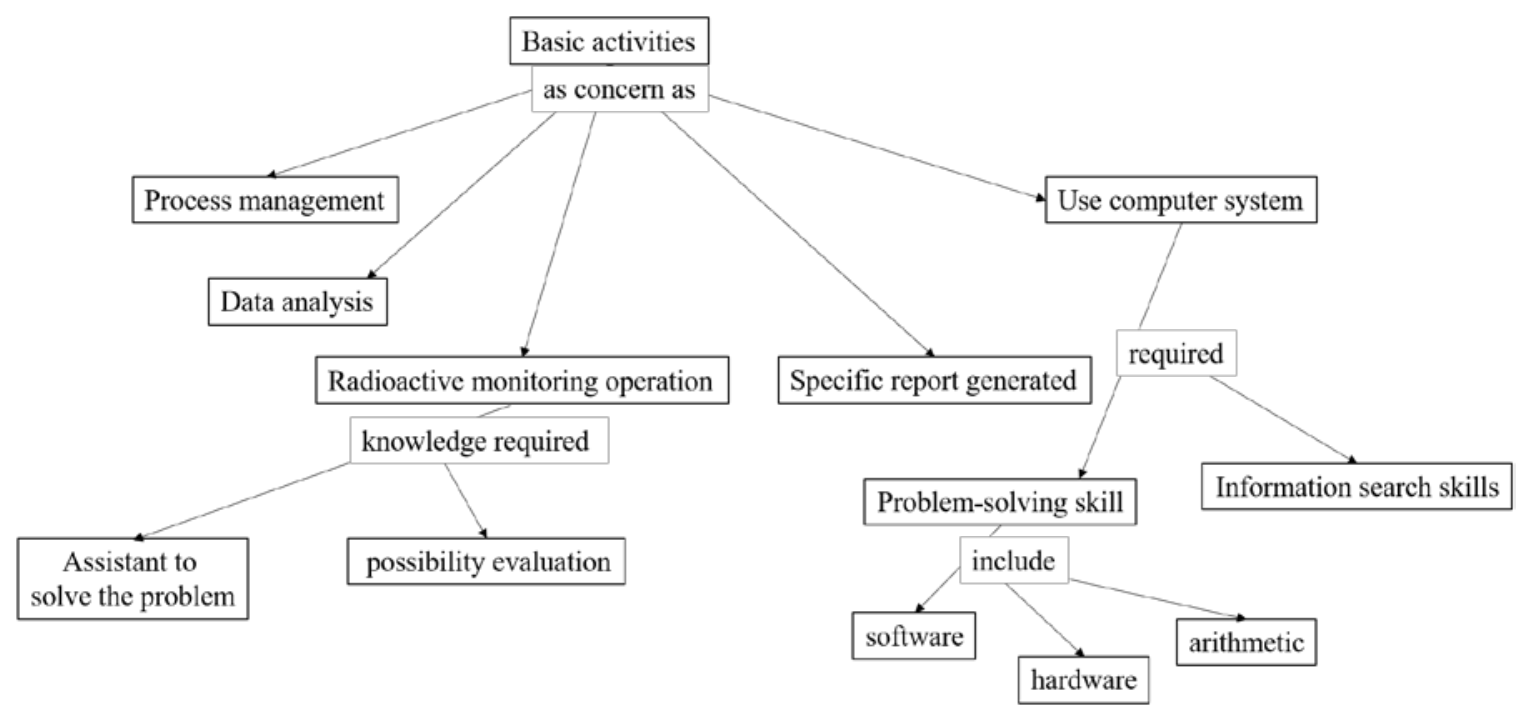

Fig.4 Acitivities diagram(one section)

Research Result. This study produced a wide range of concept map, some of the figure is traditional, with characteristics of conceptualization, but also there is a lot of are not meet researcher's expectation. Process diagram used in the highly process of knowledge field, especially it may be used to identify or describe not good edit program or process. Activity diagram seems to be useful, and it may be more specific. The process of experimental also proved that if a person tries to save what an expert's know, to lead to a range wide but insufficient knowledge model is the best way. We should pay attention to the knowledge as the results of this study. This knowledge can be used in the workers training, help workers to be qualified with skills in order to replace the retiring experts.

\section{Summary}

Expert knowledge generally refers to using empirical and sentenced to solve problem. The expert knowledge is important and necessary for any professional field, We can learn unique and effective methods and knowledge systems to heart, so it can greatly save our time in problem solving. Concept map is used to organize and represent knowledge, it can present better when extract expert knowledge system and structure which promote knowledge elicitation, and effectively guarantee the accuracy of knowledge.

\section{Acknowledgements}

This paper is one of the achievement ( "Application Research on Knowledge Visualization Technology Based on Mobile Internet”) of natural science College key project of in Anhui Sanlian University (project No kjzd2016004),also is supported by the natural science foundation of the Anhui Higher Education Institutions of China(Grant No.KJ2016A894).

\section{References}

[1] Bradley, J. H. , Paul, R. , \& Seeman, E. (2006) Analyzing the structure of expert knowledge. Information and Management 43 :p. 77.

[2] Bransford, J. D. , B rown, A. , \& Cocking, R. (2000). How peop le learn: Mind, brain, experience and school]. Washington,DC: National Academy Press: p.19.

M.A. Green: High Efficiency Silicon Solar Cells (Trans Tech Publications, Switzerland 1987).

[3] Coffey, J. W. , Hoffman, R. R. , Canas, A. J. , \& Kenneth,M. F. (2002). A concept map based knowledge modeling approach to expert knowledge sharing, IKS 20022 The I ASTED International Conference.Information on http: / /www. ihmc. us/users/acanas/Publications/ IKS2002 / IKS. html. 
[4] Coffey, J. W. , Eskridge, T. , \&Sanchez, D. ( 2004 ). A case study in knowledge elicitation for institute memory p reservation using Concept Maps. First Int Conference on Concept Mapping in 2004.

[5] Eppler, M. J. , \& Burkard, R. A. (2004). Knowledge Visualization. Towards a New Discipline and its Fields of Application, ICA Working Paper \#2 /2004. University of Lugano, Lugano.

[6] Hoffman, R. R. , Shadbolt , N . R. , Burton, A. M. , \& Klein, G. (1995). Eliciting knowledge from experts: A methodological analysis. O rganizational Behavior and Human Decision Processes, 62(2).p.129.

[7] Liu Dayou, Huang Xiangxi, , \& Zheng Fangqing. ( 1988 ). The general introduction to expert system development( in Chinese). Computer Science, (2),p.49.

[8] Xia Huosong(1999). Knowledge acquisitions of marketing expert system ( in Chinese). Journal of W uhan textile S. H. T. institute,(3) ,p.462.

[9] Johnson, P. , Zualkernan, I . , \& Garber, S. (1987) , Specification of expertise. International Journal of Man2 Machine Studies,(26) p.161.

[10] Zhao Guoqing, Huang Ronghuai, \& Lu Zhijian ( 2005). Theory and methodology of knowledge visualization ( in Chinese). Open Education Research, 11 (1) .p.23. 